\title{
COMBINATION OF AHP AND PROMETHEE FOR MEASURING QUALITY OF OBJECT ORIENTED SOFTWARE DESIGN
}

\author{
Petrus Mursanto \\ Enterprise Computing Lab (ECL) \\ Faculty of Computer Science - Universitas Indonesia \\ Kampus UI, Depok 16424, INDONESIA \\ E-mail: santo@cs.ui.ac.id \\ Arwin Halim \\ Master of Information Technology Programme \\ Faculty of Computer Science - Universitas Indonesia \\ Jl. Salemba Raya 4, Jakarta, INDONESIA \\ E-mail: win.halim@gmail.com
}

\begin{abstract}
Measuring quality of object oriented (OO) software is challenging mainly because we are facing a number of more or less dependent parameters that characterize the properties of good OO design. This paper proposes a combination of AHP and PROMETHEE for measuring object oriented software design. We have compared AHP and PROMETHEE performance for such purpose in the ISAHP 2011. The result suggested a combination of both methods for better accuracy and more robust measurement.

The proposed method combines the AHP's pairwise comparison for defining weights of criteria and employing the PROMETHEE decision aid for selecting ranks of OO software quality. This method has been applied for defining ranks over a number of object oriented software. The result shows that the integration of AHP and PROMETHEE deliver more accurate and robust measurement compared to the result of each method individually.
\end{abstract}

Keywords: AHP, PROMETHEE, object oriented, OO metrics. 


\section{Introduction}

Quality is one of the main focuses in the object oriented software development process. It is measurable and represented by several criteria which denote the degree of object oriented properties applicability within a software application. Object oriented (OO) software quality can be measured by calculating the value of metrics that show a certain quality factors, such as maintainability, reusability, understandability, etc. A systematic procedure is required for determining the most optimal software design based on existing quality factors.

\section{Literature Review}

The Analytical Hierarchy Process (AHP) is a structured technique to analyze the complex decision using pairwise comparisons. It can determine the various factor weights and factor evaluations accurately. Preference Ranking Organization Method for Enrichment Evaluation (PROMETHEE) is an outranking multiple criteria methods [1]. Both methods can be used to find out how important a criterion in relative terms when compared with other criteria [2]. Comparison of AHP and PROMETHEE performance has been evaluated in [3]. Further elaboration on the implementation of ANP for measuring OO software quality has been addressed in [4]. Considering that PROMETHEE does not support weight determination and criteria hierarchy, there are several research and literatures that combine AHP and PROMETHEE. The advantages of each method can be combined to obtain better accurate and more robust result in defining relative quality over a number of $\mathrm{OO}$ softwares. The AHP will be applied to determine the priority of the quality factor. The priority will be used by the PROMETHEE to specify which software conforms to good OO design.

\section{Hypotheses/Objectives}

The main feature of AHP's pairwise comparison can be combined with PROMETHEE's decision method for selecting quantitative metrics that represent quality of object oriented software design. The result of measurement using AHP and PROMETHEE will be proven more accurate compared to the result of applying AHP or PROMETHEE independently.

\section{Research Design/Methodology}

The experiment for calculation the PROMETHEE utilizes the D-Sight to determine the best design from alternative designs. In the first stage, the criteria of AHP method will be included as criteria in PROMOTHEE. The next step is to determine the expected metrics value, maximum or minimum, for each criterion in accordance with reference PROMOTHEE's references. Based on the aggregated preference indices for each design, we can calculate the net outranking flow and select the most suitable design.

A set of experiments were conducted upon the same software set. Weight set produced by AHP's pairwise comparison is used as default weights which are alterable during experiments. Default preference function for all criteria is type 3 (linear) and each criteria standard deviation is used as threshold. Since AHP does not support minimum-maximum setting, this parameter is set to maximum for all criteria. An experimental tool has been 
developed with the possibility to alter weight criteria groups (encapsulation, inheritance, polymorphism, and coupling).

\section{Data/Model Analysis}

There are many OO metrics have been developed that describe the applicability of good OO properties within a software. Two most popular sets are utilized in this paper, i.e. Metrics for Object Oriented Software Engineeering (MOOSE) [5] and Metrics for Object Oriented Design (MOOD) [6]. Hierarchical structure of AHP criteria is shown for MOOSE (Fig 1a) and MOOD (Fig 2b).

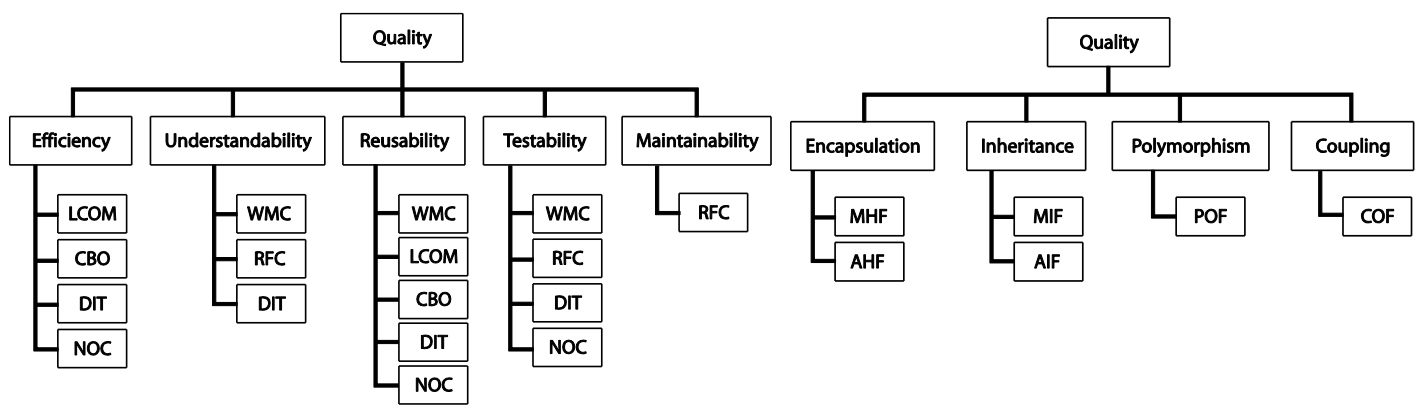

Fig 1a. MOOSE hierarchical criteria

Fig 1b. MOOD hierarchical MOOD

PROMETHEE I or partial ranking is achieved by computing alternatives' strength and weakness. Figure 2a depicts an example of PROMETHEE I diagram. PROMETHEE II or complete ranking is achieved by calculating net flow for each of the alternatives by reducing weakness from its strength. Figure $2 \mathrm{~b}$ describes PROMETHEE II diagram for corresponding partial ranks in Figure 1.

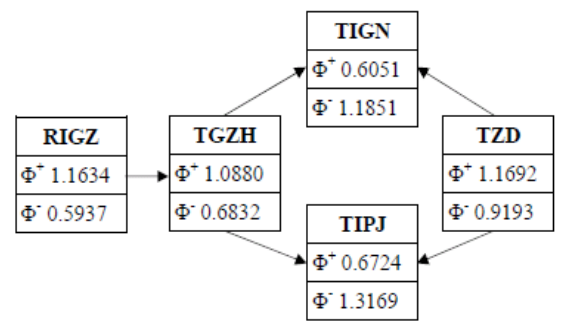

Fig 2a. PROMETHEE I

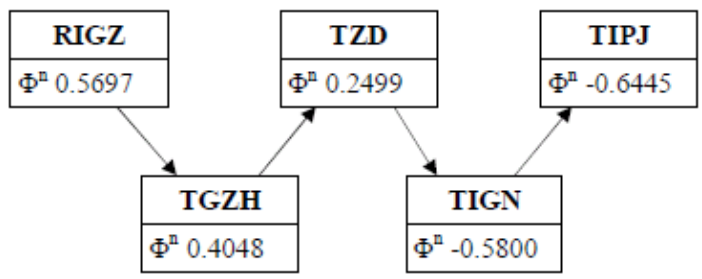

Fig 2b. PROMETHEE II

\section{Limitations}

The proposed method has been verified for a limited number of OO software which are part of $\mathrm{OO}$ development library. Such library components are designed for high reusability and hence embed the most complete features. In fact only part of the available features are utilized in operational software system. Therefore, the applicability of OO properties in software component library differs from the ones possessed by customized component with limited resources such as in mobile platform. 


\section{Conclusions}

This paper proposes a systematic approach in determining relative quality over a number of OO software design. This approach has combined AHP and PROMETHEE method to specify the design. The main assessments in determining the best design are a clear criterion and metric values of the alternative design. In our experiment, this step is performed by AHP. Using PROMETHEE, we produce quantitative values to represent software design quality, relatively one among the others. These values can be used to rank quality among the softwares being evaluated.

\section{Key References}

[1] J.P. Brans and B. Mareschal, "PROMETHEE Methods", https://www. inf.unideb.hu/valseg/dolgozok/anett.racz/docs/ DSS/Promethee.pdf

[2] F. Janos, "Introduction to Decision Making Methods", Laboratory of Operations Research and Decision Systems, Computer and Automation Institute, Hungarian Academy of Sciences, 2005.

[3] Mursanto, P., Wida Sari (2011). Defining Relative Qualities of Object Oriented Design Implementation Using AHP and PROMETHEE. ISAHP, Sorrento - Italy.

[4] Mursanto, P. (2013) Accuracy Improvement of Object Oriented Software Design Measurement Using Analytic Network Process. ISAHP, Kuala Lumpur - Malaysia.

[5] Chidamber, Shyam, Kemerer, Chris F (1993) A Metrics Suite for Object-Oriented Design, M.I.T. Sloan School of Management E53-315.

[6] Abrue F.B., Esteves R., Goulao M. (1996) The Design of Eiffel Programs: Quantitative Evaluation Using the MOOD Metrics, Proceeding of TOOLS, USA. 